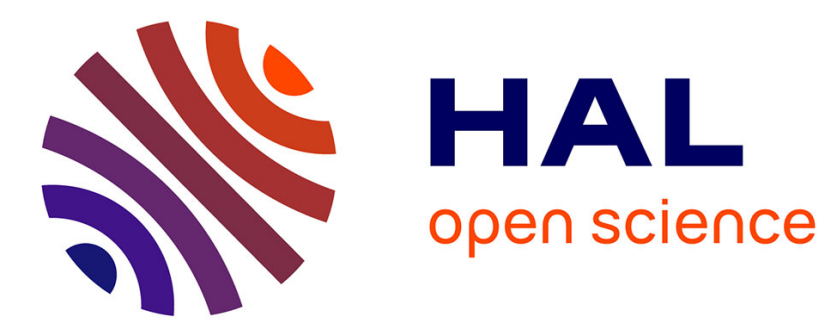

\title{
Benchmarking IPOP-CMA-ES-TPA and IPOP-CMA-ES-MSR on the BBOB Noiseless Testbed
}

\author{
Asma Atamna
}

\section{To cite this version:}

Asma Atamna. Benchmarking IPOP-CMA-ES-TPA and IPOP-CMA-ES-MSR on the BBOB Noiseless Testbed. GECCO (Companion), workshop on Black-Box Optimization Benchmarking (BBOB'2015), Jul 2015, Madrid, Spain. , 10.1145/2739482.2768467 . hal-01323506

\section{HAL Id: hal-01323506 https://hal.inria.fr/hal-01323506}

Submitted on 30 May 2016

HAL is a multi-disciplinary open access archive for the deposit and dissemination of scientific research documents, whether they are published or not. The documents may come from teaching and research institutions in France or abroad, or from public or private research centers.
L'archive ouverte pluridisciplinaire HAL, est destinée au dépôt et à la diffusion de documents scientifiques de niveau recherche, publiés ou non, émanant des établissements d'enseignement et de recherche français ou étrangers, des laboratoires publics ou privés. 


\title{
Benchmarking IPOP-CMA-ES-TPA and IPOP-CMA-ES-MSR on the BBOB Noiseless Testbed
}

\author{
Asma Atamna \\ Projet TAO, Inria \\ Saclay-Île-de-France \\ LRI, Bât. 660, Univ. Paris-Sud \\ 91405 Orsay Cedex, France \\ atamna@Iri.fr
}

\begin{abstract}
We benchmark IPOP-CMA-ES, a restart Covariance Matrix Adaptation Evolution Strategy with increasing population size, with two step-size adaptation mechanisms, TwoPoint Step-Size Adapation (TPA) and Median Success Rule (MSR), on the BBOB noiseless testbed. We then compare IPOP-CMA-ES-TPA and IPOP-CMA-ES-MSR to IPOPCMA-ES with the standard step-size adaptation mechanism, Cumulative Step-size Adaptation (CSA). We conduct experiments for a budget of $10^{5}$ times the dimension of the search space. As expected, the algorithms perform alike on most functions. However, we observe some relevant differences, the most significant being on the attractive sector function where IPOP-CMA-TPA and IPOP-CMA-CSA outperform IPOP-CMA-MSR, and on the Rastrigin function where IPOP-CMA-MSR is the only algorithm to solve the function in all tested dimensions. We also observe that at least one of the three algorithms is comparable to the best BBOB-09 artificial algorithm on 13 functions.
\end{abstract}

\section{Categories and Subject Descriptors}

G.1.6 [Numerical Analysis]: Optimization-global optimization, unconstrained optimization; F.2.1 [Analysis of Algorithms and Problem Complexity]: Numerical Algorithms and Problems

\section{Keywords}

Benchmarking, Black-box optimization

\section{INTRODUCTION}

This paper compares three step-size adaptation methods coupled with IPOP-CMA-ES [2], a restarted version of the state-of-the-art Evolution Strategy (ES), the Covariance Matrix Adaptation Evolution Strategy (CMA-ES) [8], where the population size is increased for each restart, on the

Permission to make digital or hard copies of all or part of this work for personal or classroom use is granted without fee provided that copies are not made or distributed for profit or commercial advantage and that copies bear this notice and the full citation on the first page. Copyrights for components of this work owned by others than the author(s) must be honored. Abstracting with credit is permitted. To copy otherwise, or republish, to post on servers or to redistribute to lists, requires prior specific permission and/or a fee. Request permissions from permissions@ acm.org.

GECCO '15, July 11 - 15, 2015, Madrid, Spain

(C) 2015 Copyright held by the owner/author(s). Publication rights licensed to ACM. ISBN 978-1-4503-3488-4/15/07 . \$ \$15.00

DOI: http://dx.doi.org/10.1145/2739482.2768467
BBOB noiseless testbed $[3,7]$. The step-size adaptation algorithms under consideration are Two-Point Step-Size Adaptation (TPA) [5], Median Success Rule (MSR) [1], and Cumulative Step-Size Adaptation (CSA) [8], the latter being the default step-size adaptation method in CMA-ES. We first recall the general principle of the considered ES, we then describe the studied step-size adaptation algorithms, with a particular focus on TPA and MSR, and evaluate them empirically.

\section{THE $(\mu / \mu, \lambda)$-ES}

In this paper, we consider the $(\mu / \mu, \lambda)$-ES with weighted recombination, where $\lambda$ is the population size, $\mu$ is the number of parents, and ',' denotes non-elitist selection [4]. At iteration $t, \lambda$ offspring, $\mathbf{X}_{t}^{1}, \ldots, \mathbf{X}_{t}^{\lambda}$, are sampled independently from a multivariate normal distribution according to

$$
\mathbf{X}_{t}^{i}=\mathbf{X}_{t}+\sigma_{t} \mathcal{N}_{t}\left(0, \mathbf{C}_{t}\right), i=1, \ldots, \lambda
$$

where $\mathcal{N}_{t}\left(0, \mathbf{C}_{t}\right)$ is the multivariate normal distribution with mean 0 and covariance matrix $\mathbf{C}_{t}, \sigma_{t}$ is the step-size and defines the width of the sampling distribution. The $\mu$ best offspring are recombined to form the new solution

$$
\mathbf{X}_{t+1}=\sum_{i=1}^{\mu} w_{i} \mathbf{X}_{t}^{i: \lambda}
$$

where $\mathbf{X}_{t}^{i: \lambda}$ is the $i$ th best offspring fitness-wise, $w_{i}>0$ and $\sum_{i=1}^{\mu} w_{i}=1$. In adaptive ES, $\sigma_{t}$ and $\mathbf{C}_{t}$ are updated during the search process in order to achieve fast convergence.

\section{IPOP-CMA-ES}

IPOP-CMA-ES consists in launching independent restarts of CMA-ES by increasing the population size by a factor of two for each restart. Increasing the population size allows for a better covering of the search space and improves the performance of CMA-ES on multimodal functions [2]. The principle of the algorithm can be summed up in two steps:

\section{1. run CMA-ES}

2. if CMA-ES stops before reaching the target value and before exceeding the budget, double the population size and go to step 1

For a detailed description of the algorithm, see [2].

$C M A-E S$. In this paper, we consider the $(\mu / \mu, \lambda)$-CMA-ES with weighted recombination, fully described in [8]. 


\section{STEP-SIZE ADAPTATION METHODS}

This section describes the three step-size adaptation methods under investigation.

\subsection{TPA}

In Two-Point Step-Size Adaptation, the first two offspring are sampled along the shift vector from the previous solution, $\mathbf{X}_{t-1}$, to the current solution $\mathbf{X}_{t}$, as a mirrored pair, symmetric to $\mathbf{X}_{t}$.

$$
\mathbf{X}_{t}^{1,2}=\mathbf{X}_{t} \pm \sigma_{t} \times\left\|\mathcal{N}_{t}(0, \mathbf{I})\right\| \frac{\mathbf{X}_{t}-\mathbf{X}_{t-1}}{\left\|\mathbf{X}_{t}-\mathbf{X}_{t-1}\right\|}
$$

where $\mathbf{I}$ is the identity matrix. We decide whether to increase or decrease the step-size $\sigma_{t}$ depending on the fitness of $\mathbf{X}_{t}^{1}$ and $\mathbf{X}_{t}^{2}$ : if $\mathbf{X}_{t}^{1}$ is better than $\mathbf{X}_{t}^{2}, \sigma_{t}$ is increased as this indicates that there are better solutions in the direction of the latest solution shift. Otherwise, it is decreased. The following equations give the step-size update.

$$
\begin{gathered}
s_{1}=\left(1-c_{\sigma}\right) s_{t-1}+c_{\sigma} \frac{\operatorname{rank}\left(\mathbf{X}_{t}^{2}\right)-\operatorname{rank}\left(\mathbf{X}_{t}^{1}\right)}{\lambda-1} \\
\sigma_{t+1}=\sigma_{t} \exp \left(\frac{s_{t}}{d_{\sigma}}\right)
\end{gathered}
$$

where $\operatorname{rank}\left(\mathbf{X}_{t}^{i}\right)$ is the fitness ranking of the $i$ th individual among the entire population, $s_{0}=0, c_{\sigma}=0.3$, and $d_{\sigma}=$ $\sqrt{D}$ where $D$ is the dimension of the search space. A more thorough description of the algorithm can be found in [5].

\subsection{MSR}

The Median Success Rule Step-Size Adaptation can be seen as a generalization of the $1 / 5$ th success rule [10] to the case of $(\mu / \mu, \lambda)$-ES. The success is defined as the median individual (fitness-wise) of the current population, $\mathbf{X}_{t}^{m(\lambda)}$, being better than the $j$ th best individual of the previous population, $\mathbf{X}_{t-1}^{j: \lambda}$. In practice, $j$ is chosen such that the median success probability is $1 / 2$ with optimal step-size on the sphere function [1]. The idea is then to increase the stepsize if $\mathbf{X}_{t}^{m(\lambda)}$ is fitter than $\mathbf{X}_{t-1}^{j: \lambda}$ and decrease it otherwise. The step-size $\sigma_{t}$ is updated as

$$
\begin{gathered}
s_{1}=\left(1-c_{\sigma}\right) s_{t-1}+c_{\sigma} \frac{2}{\lambda}\left(K_{\text {succ }}-\frac{\lambda}{2}\right) \\
\sigma_{t+1}=\sigma_{t} \exp \left(\frac{s_{t}}{d_{\sigma}}\right)
\end{gathered}
$$

where $K_{\text {succ }}$ is the number of successful individuals, $s_{0}=0$, $c_{\sigma}=0.3$, and $d_{\sigma}=2-2 / D$.

\subsection{CSA}

The Cumulative Step-Size Adaptation is the standard stepsize adaptation method in CMA-ES. A detailed description of the method can be found in [8].

\section{EXPERIMENTAL PROCEDURE}

We ran the algorithms with a budget of $10^{5} \times D$ on the $\mathrm{BBOB}$ noiseless functions in six different dimensions. We used the python implementation of CMA-ES, cma 1.1.06. The source code can be found at [11]. TPA, MSR, and CSA are implemented in cma 1.1.06 as well as the IPOP restart strategy. For each run of the algorithms, the initial solution $\mathbf{X}_{0}$ is sampled uniformly in $[-4,4]^{D}$ and the initial step-size $\sigma_{0}$ is set to 2.5. The maximum number of restarts is set to 9 . For all other parameters, default values are used (for instance, the population size $\lambda=4+\lfloor 3 \ln D\rfloor$ and the number of parents $\mu=\lambda / 2$ ).

\section{RESULTS}

Results from experiments according to [6] on the benchmark functions given in $[3,7]$ are presented in Figures 1, 3 and 4 and in Tables 1 and 2. The expected running time (ERT), used in the figures and tables, depends on a given target function value, $f_{\mathrm{t}}=f_{\mathrm{opt}}+\Delta f$, and is computed over all relevant trials as the number of function evaluations executed during each trial while the best function value did not reach $f_{\mathrm{t}}$, summed over all trials and divided by the number of trials that actually reached $f_{\mathrm{t}}[6,9]$. Statistical significance is tested with the rank-sum test for a given target $\Delta f_{\mathrm{t}}$ using, for each trial, either the number of needed function evaluations to reach $\Delta f_{\mathrm{t}}$ (inverted and multiplied by -1 ), or, if the target was not reached, the best $\Delta f$-value achieved, measured only up to the smallest number of overall function evaluations for any unsuccessful trial under consideration.

For the sake of simplicity, we will refer to IPOP-CMA-ESTPA, IPOP-CMA-ES-MSR, and IPOP-CMA-ES-CSA as TPA, MSR, and CSA respectively in the following.

ERT versus dimension. Figure 1 shows that in $5-D$ (respectively 20-D), TPA, MSR, and CSA solve 22 (respectively 19), 20 (respectively 20), and 22 (respectively 20) out of 24 functions. For unsolved functions (mainly multi-modal and weakly structured multi-modal functions), a larger budget is required (at least $10^{6} \times D$ function evaluations). The algorithms have a comparable performance on most of the functions and scale similarly with the dimension. This corresponds to our expectations, as the three algorithms are very similar. On some functions, however, we observe relevant differences in the performance: on function 1 (sphere), TPA performs significantly better than MSR and CSA in at least one dimension. We also observe a significant difference on function 6 (attractive sector) where TPA and CSA outperform MSR in large dimensions. Single runs on function 6 show that MSR generates smaller step-sizes than TPA and CSA, which leads to its larger ERT. Figure 2 displays single runs of MSR (left) and CSA (right) in 20-D (due to space limitations, results for TPA are not presented). On function 3 (separable Rastrigin), MSR has the best performance. Our explanation is that having small step-sizes avoids getting stuck in local optima. On functions 16 (Weierstrass) and 19 (Griewank-Rosenbrock), TPA and CSA perform very similarly and better than MSR. On function 20 (Schwefel), CSA performs slightly better than TPA in small dimensions. The gap we see in 10- $D$ between TPA and CSA is due to insufficient budget and should disappear by increasing the budget. Another significant difference is observed on function 23 (Katsuuras) where MSR solves the function within the maximum budget and performs better than TPA and CSA. On function 21 (Gallagher 101 peaks), a larger budget is necessary to decide whether the observed difference is significant, since the ERTs are close to the maximum budget. Another observation is that each algorithm performs similarly on the original/rotated ellipsoid and Rosenbrock due to their rotational invariance. On Rastrigin functions, however, this is not the case, likely because the rotated function does not correspond to the original one. 

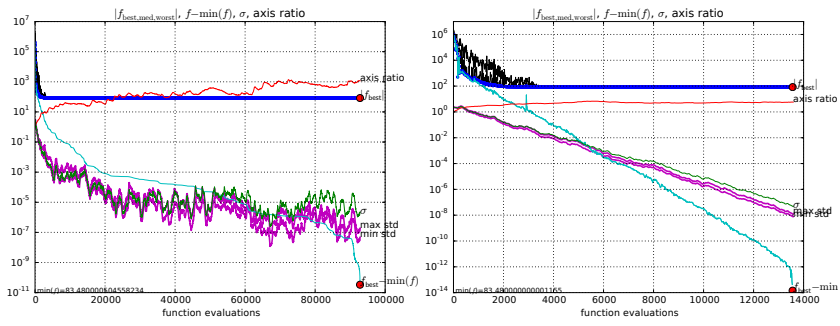

Figure 2: Single runs of IPOP-CMA-MSR (left) and IPOP-CMA-CSA (right) on one instance of the attractive sector function in $20-D$. $x$-axis shows function evaluations. Line with dots (blue): best $f$-value of the iteration in absolute value, median and worst displayed in thin black lines; cyan line: difference between current $f$-value and $f_{\text {opt }}$; green line: stepsize $\sigma_{t}$, largest and smallest coordinate-wise standard deviation of the sample distribution in purple; red line: square root of the condition number of the covariance matrix.

Empirical cumulative distribution functions. Figures 3 and 4 show the empirical cumulative distribution functions (ECDFs) of the number of function evaluations for 50 targets in dimensions 5 and 20 respectively. In $5-D$, the ECDFs are quite similar for moderate and ill-conditioned functions. On separable functions, MSR solves about $82 \%$ of the problems for the fixed budget $\left(10^{5} \times D\right)$ while TPA and CSA solve about $73 \%$. On multi-modal functions, TPA and CSA manage to solve all problems while MSR solves about $88 \%$ of the problems. While no algorithm solves all weakly structured multi-modal problems, TPA and CSA solve up to $76 \%$ of the problems for the maximum budget while MSR only solves about $56 \%$. On the overall set of functions, TPA, MSR, and CSA solve roughly the same proportion of problems up to $10^{4} \times D$ function evaluations. For the maximum budget, however, TPA and CSA solve about $90 \%$ of the problems while MSR only solves about $84 \%$. In $20-D$, two main differences are observed: firstly, TPA and CSA solve about $8 \%$ (respectively 10\%) less separable (respectively multi-modal) problems than in 5- $D$ (none of them managed to solve function 3 in 20-D). Secondly, CSA is better than MSR and TPA on weakly structured multi-modal problems and solves about $50 \%$ of the problems, being $10 \%$ more than MSR and $13 \%$ more than TPA.

\section{DISCUSSION}

We evaluated IPOP-CMA-ES with two different and relatively new step-size adaptation schemes, TPA and MSR, on the BBOB noiseless continuous functions. We then compared them to IPOP-CMA-ES with the standard step-size adaptation method, CSA. As expected, empirical results showed that the three algorithms need nearly the same number of function evaluations in average to solve the target $f_{\mathrm{t}}=f_{\mathrm{opt}}+10^{-8}$ on a large number of functions. However, significant differences were observed, the most notable were on the attractive sector function where TPA and CSA outperformed MSR in large dimensions and on Rastrigin where MSR was the best. 16 functions out of 24 were solved by all the algorithms in all dimensions while some multi-modal and weekly structured multi-modal functions remained unsolved because the chosen budget $\left(10^{5} \times D\right.$ function evaluations $)$ was insufficient. On the other hand, the performance was comparable to the best BBOB-09 results on 13 functions for at least one algorithm, generally in large dimensions.

\section{Acknowledgments}

This work was supported by the NumBBO project (grant ANR-2012-MONU-0009) of the French National Research Agency. The author would like to thank Nikolaus Hansen and Anne Auger for their comments on this work.

\section{REFERENCES}

[1] O. Ait Elhara, A. Auger, and N. Hansen. A median success rule for non-elitist evolution strategies: Study of feasibility. In Genetic and Evolutionary Computation Conference (GECCO 2013), Proceedings, pages 415-422. ACM, 2013.

[2] A. Auger and N. Hansen. A restart CMA evolution strategy with increasing population size. In Proceedings of the IEEE Congress on Evolutionary Computation (CEC 2005), pages 1769-1776. IEEE Press, 2005.

[3] S. Finck, N. Hansen, R. Ros, and A. Auger. Real-parameter black-box optimization benchmarking 2009: Presentation of the noiseless functions. Technical Report 2009/20, Research Center PPE, 2009. Updated February 2010.

[4] N. Hansen, D. Arnold, and A. Auger. Evolution strategies. To appear in Janusz Kacprzyk and Witold Pedrycz (Eds.): Handbook of Computational Intelligence, Springer.

[5] N. Hansen, A. Atamna, and A. Auger. How to assess step-size adaptation mechanisms in randomised search. In Parallel Problem Solving from Nature PPSN XIII, Proceedings, pages 60-69. Springer, 2014.

[6] N. Hansen, A. Auger, S. Finck, and R. Ros. Real-parameter black-box optimization benchmarking 2012: Experimental setup. Technical report, INRIA, 2012.

[7] N. Hansen, S. Finck, R. Ros, and A. Auger. Real-parameter black-box optimization benchmarking 2009: Noiseless functions definitions. Technical Report RR-6829, INRIA, 2009. Updated February 2010.

[8] N. Hansen and A. Ostermeier. Completely derandomized self-adaptation in evolution strategies. Evolutionary Computation, 9(2):159-195, 2001.

[9] K. Price. Differential evolution vs. the functions of the second ICEO. In Proceedings of the IEEE International Congress on Evolutionary Computation, pages 153-157, 1997.

[10] I. Rechenberg. Evolutionsstrategie '94. Frommann-Holzboog Verlag, 1994.

[11] http://coco.gforge.inria.fr/doku.php?id=bbob-2015-results. 

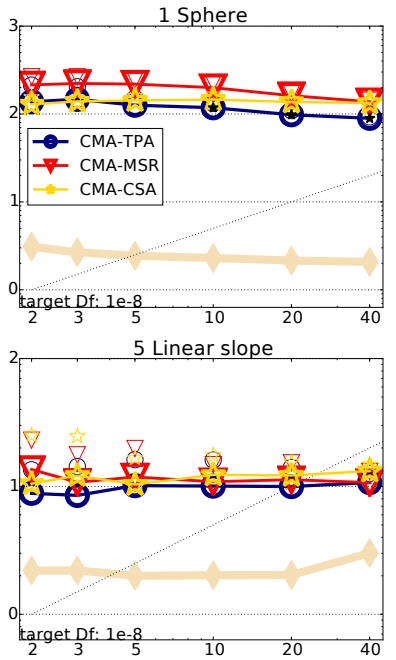

9 Rosenbrock rotated
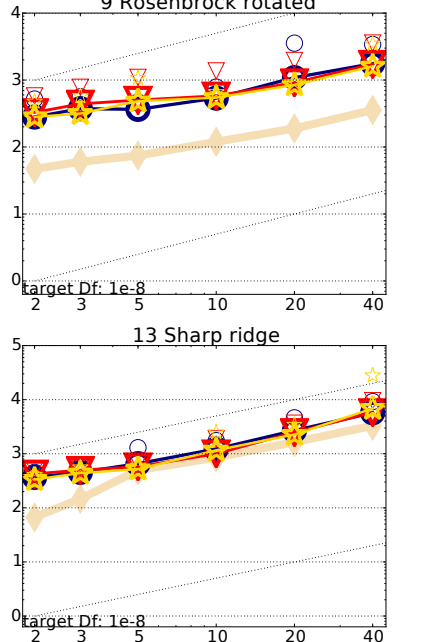

17 Schaffer F7, condition 10
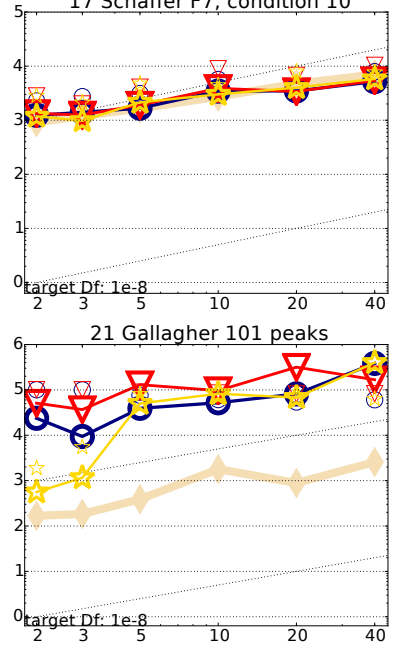
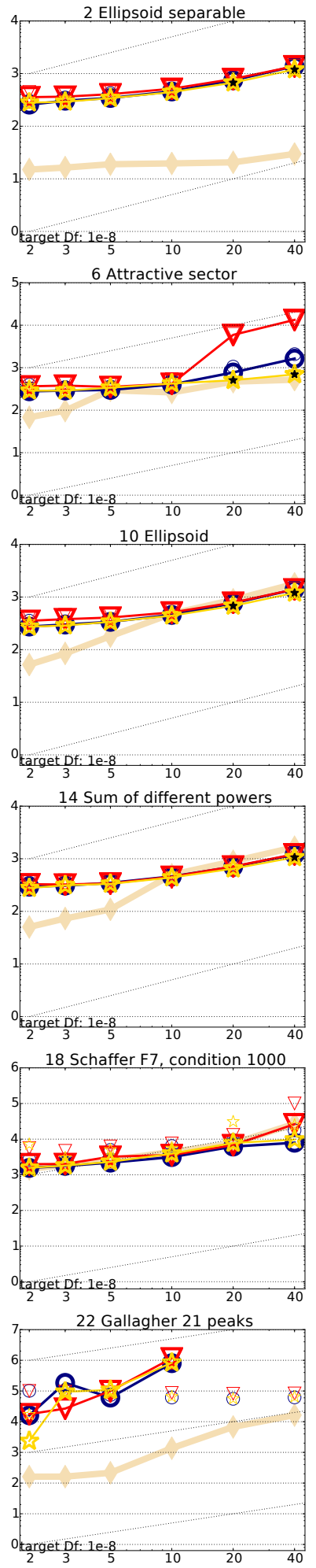
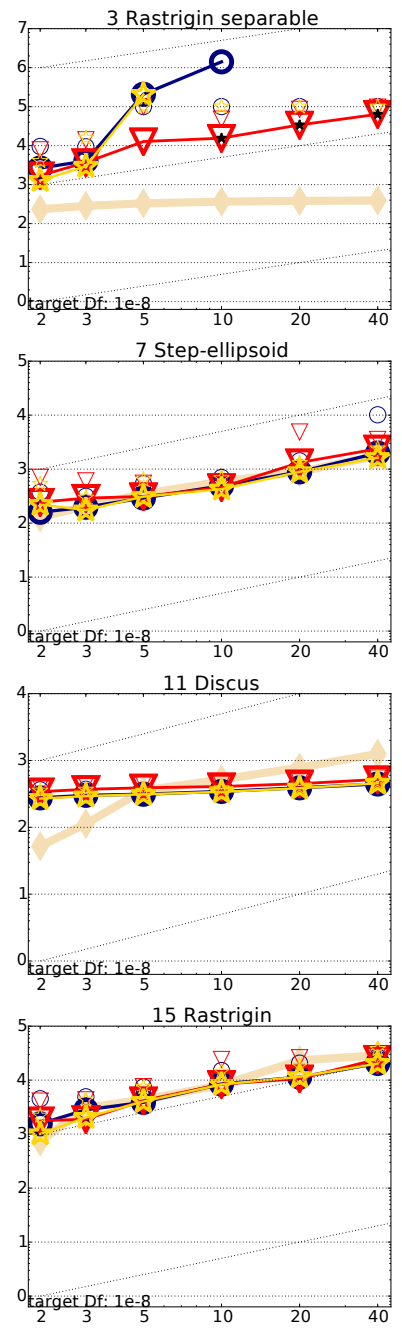

7 , 19 Griewank-Rosenbrock F8F2
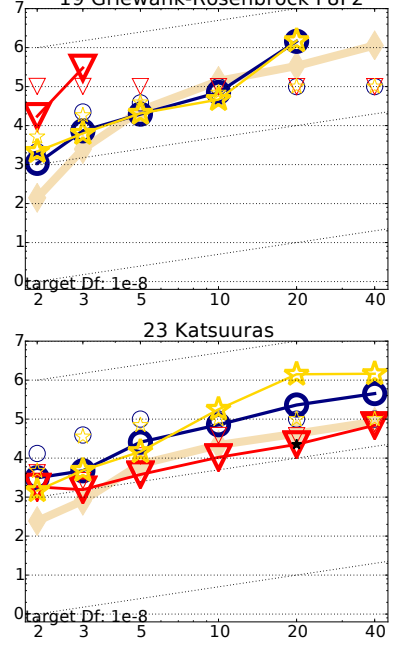
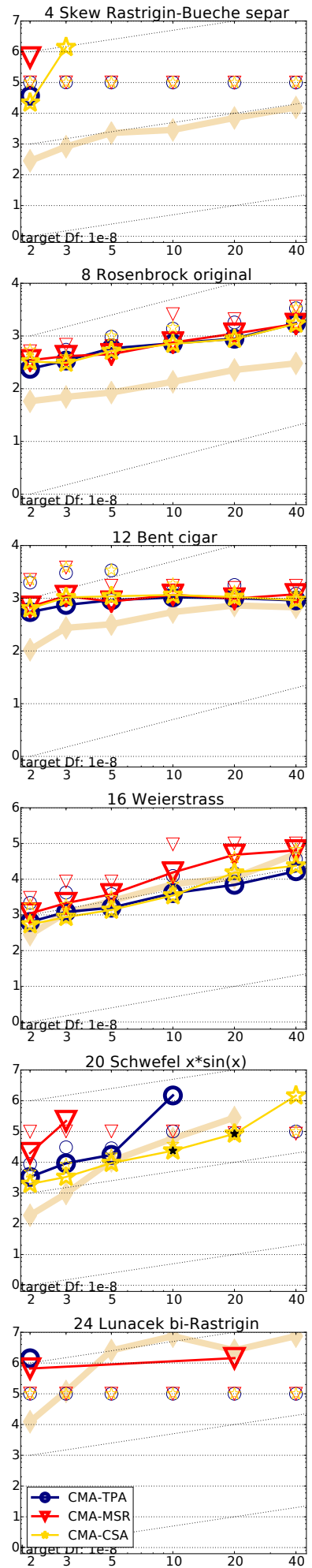

Figure 1: Expected running time (ERT in number of $f$-evaluations as $\log _{10}$ value), divided by dimension for target function value $10^{-8}$ versus dimension. Slanted grid lines indicate quadratic scaling with the dimension. Different symbols correspond to different algorithms given in the legend of $f_{1}$ and $f_{24}$. Light symbols give the maximum number of function evaluations from the longest trial divided by dimension. Black stars indicate a statistically better result compared to all other algorithms with $p<0.01$ and Bonferroni correction number of dimensions (six). Legend: $\circ$ :CMA-TPA, $\nabla:$ CMA-MSR, :CMA-CSA 



Figure 3: Bootstrapped empirical cumulative distribution of the number of objective function evaluations divided by dimension (FEvals/DIM) for 50 targets in $10^{[-8.2]}$ for all functions and subgroups in 5-D. The "best 2009" line corresponds to the best ERT observed during BBOB 2009 for each single target. 

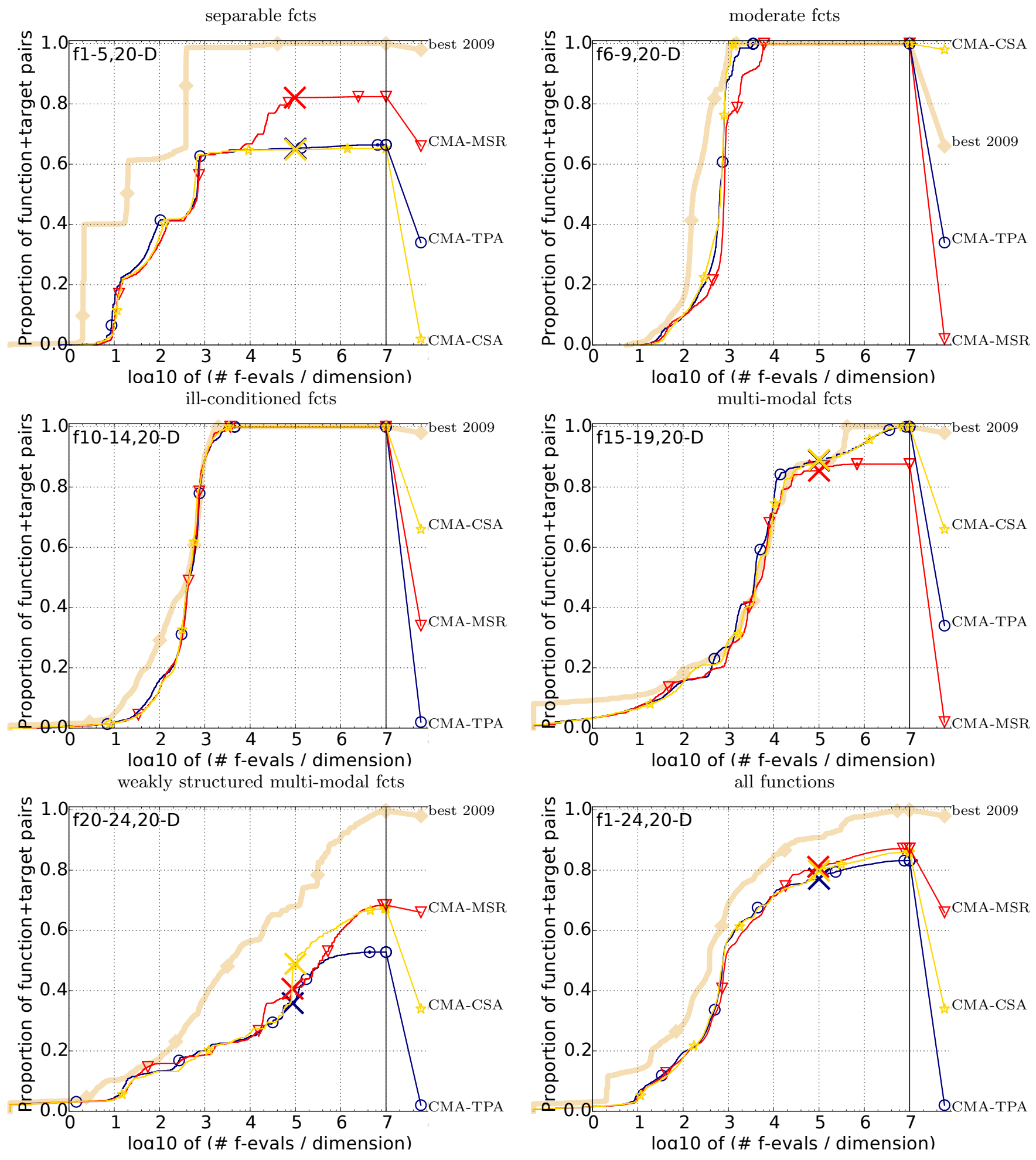

Figure 4: Bootstrapped empirical cumulative distribution of the number of objective function evaluations divided by dimension (FEvals/DIM) for 50 targets in $10^{[-8 . .2]}$ for all functions and subgroups in 20-D. The "best 2009" line corresponds to the best ERT observed during BBOB 2009 for each single target. 


\begin{tabular}{|c|c|c|c|c|c|c|c|c|c|c|c|c|c|c|c|c|c|}
\hline$\Delta f_{\text {opt }}$ & $1 \mathrm{e} 1$ & $1 \mathrm{e} 0$ & $1 \mathrm{e}-1$ & $1 \mathrm{e}-2$ & $1 \mathrm{e}-3$ & $1 \mathrm{e}-5$ & $1 \mathrm{e}-7$ & \#succ & $\Delta f_{\mathrm{opt}}$ & $1 \mathrm{e} 1$ & $1 \mathrm{e} 0$ & $1 \mathrm{e}-1$ & $1 \mathrm{e}-2$ & $1 \mathrm{e}-3$ & $1 e-5$ & $1 e-7$ & \#succ \\
\hline f1 & 11 & 12 & 12 & 12 & 12 & 12 & 12 & $15 / 15$ & f13 & 132 & 195 & 250 & 319 & 1310 & 1752 & 2255 & $15 / 15$ \\
\hline AA-TH & $3.2(2)$ & $9.2(4)$ & $14(6)$ & $(5)$ & $4(4)$ & $6(4)$ & $7(8)$ & $15 / 15$ & MA-T & $2.9(0.9)$ & $3.8(1)$ & $4.2(2)$ & $4.0(1)$ & $1.2(0.2)$ & $1.3(0.4)$ & $1.2(0.2)$ & $15 / 15$ \\
\hline $\mathrm{R}$ & $3.6(4)$ & $12(3)$ & $1(5)$ & (2) & $1(6)$ & $2(9)$ & $2(6)$ & $5 / 15$ & MA & $3.2(0.5)$ & $3.6(0.7)$ & $\mathbf{3 . 8}(0.7)$ & $4.0(0.7)$ & $1.2(0.1)$ & 1.2 & 1.1 & $15 / 15$ \\
\hline MA-CSA & $3.8(3)$ & $10(4)$ & $16(3)$ & $22(4)$ & $28(3)$ & $40(3)$ & $52(5)$ & $15 / 15$ & $\mathrm{MA}-\mathrm{CSA}$ & $3.3(0.8)$ & $3.4(2)$ & $4.1(1)$ & $\mathbf{3 . 9}(0.9)$ & $1.1(0.2)$ & $\mathbf{1 . 1}(0.2)$ & $1.1(0.2)$ & $15 / 15$ \\
\hline$\Delta f_{\mathrm{opt}}$ & $1 \mathrm{e} 1$ & $1 \mathrm{e} 0$ & $1 \mathrm{e}-1$ & $1 \mathrm{e}-2$ & $1 \mathrm{e}-3$ & $1 \mathrm{e}-5$ & $1 \mathrm{e}-7$ & \#succ & $\Delta f_{\text {OF }}$ & $1 \mathrm{e} 1$ & $1 \mathrm{e} 0$ & $1 \mathrm{e}-1$ & $1 \mathrm{e}-2$ & $1 \mathrm{e}-3$ & $1 e-5$ & $1 \mathrm{e}-7$ & \#succ \\
\hline f2 & 83 & 87 & 88 & 89 & 90 & 92 & 94 & $15 / 15$ & f14 & 10 & 41 & 58 & 90 & 139 & 251 & 476 & $15 / 15$ \\
\hline IA-T & $10(2)$ & $12(2)$ & $4(0.5)$ & $5(3)$ & $15(2)$ & $17(3)$ & $18(3)$ & $5 / 15$ & MA-T & $2.1(1)$ & $3.3(2)$ & $3.7(1)$ & $3.9(1)$ & $3.9(0.9)$ & $4.0(0.5)$ & $3.1(0.5)$ & $15 / 15$ \\
\hline $\mathrm{R}$ & $12(3)$ & $3(2)$ & (2) & $5(3)$ & $16(2)$ & $8(2)$ & & $15 / 15$ & & & $3.4(2)$ & $4.7(0.7)$ & $5.0(1)$ & $4.4(\mathrm{C}$ & 4.1( & $3.1(\mathrm{C}$ & $15 / 15$ \\
\hline MA-CS & $11(2)$ & $13(2)$ & $14(1)$ & $14(2)$ & $15(1)$ & $16(1)$ & $17(2)$ & $15 / 15$ & MA & $1.7(2)$ & $2.7(1)$ & $\mathbf{3 . 6}(0.8)$ & $3.7(0.8)$ & $3.8(0.7)$ & $\mathbf{3 . 9}(0.6)$ & $\mathbf{3 . 0}(0.3)$ & $15 / 15$ \\
\hline$\Delta f_{\mathrm{opt}}$ & $1 \mathrm{e} 1$ & $1 \mathrm{e} 0$ & $1 e-1$ & $1 e-2$ & $1 e-3$ & $1 e-5$ & $1 e-7$ & \#succ & $\Delta f_{\mathrm{opt}}$ & $1 \mathrm{e} 1$ & $1 \mathrm{e} 0$ & $1 \mathrm{e}-1$ & $1 \mathrm{e}-2$ & $1 e-3$ & $1 e-5$ & $1 e-7$ & \#succ \\
\hline f3 & 716 & 1622 & 1637 & 1642 & 1646 & 1650 & 1654 & $15 / 15$ & f15 & 511 & 9310 & 19369 & 19743 & 20073 & 20769 & 21359 & $15 / 15$ \\
\hline MA-T & $0.81(0.7$ & 7)9.3(10)6 & $632(925)$ & $630(1153) 6$ & $629(926)$ & $28(766)$ & $627(458)$ & $5 / 15$ & $\mathrm{MA}$ & $1.9(2)$ & $\mathbf{0 . 9 0}(0.5)$ & $\mathbf{0 . 8 7}(0.6)$ & $\mathbf{0 . 8 8}(0.6)$ & $0.88(0.7)$ & 0.8 & & ) $15 / 15$ \\
\hline MA-1 & $1.7(2)$ & $5.7(2)$ & $36(86)$ & $\mathbf{3 6}(154)$ & $\mathbf{3 6}(155)$ & $37(164)$ & $38(12)$ & $14 / 15$ & $\mathrm{MA}$ & $1.9(2)$ & $0.95(0.8)$ & $0.89(0.7)$ & $0.89(0.6)$ & $0.91(0.6)$ & 0.9 & 0.5 & $15 / 15$ \\
\hline & $\mid 1.4(1)$ & $32(82)$ & $623(1075) 6$ & $622(460) 6$ & $621(837)$ & $619(840)$ & $618(607)$ & $5 / 15$ & (1) & $\mathbf{1 . 1 ( 0 . 9 )}$ & $1.1(0.8)$ & $0.91(0.3)$ & $0.92(0.4)$ & $0.92(0$ & 0.9 & & $15 / 15$ \\
\hline$\Delta f_{\mathrm{opt}}$ & $1 \mathrm{e} 1$ & $1 \mathrm{e} 0$ & $1 \mathrm{e}-1$ & $1 \mathrm{e}-2$ & $1 e-3$ & $1 e-5$ & $1 \mathrm{e}-7$ & \#succ & $\Delta f_{\text {opt }}$ & $1 \mathrm{e} 1$ & $1 \mathrm{e} 0$ & $1 \mathrm{e}-1$ & $1 \mathrm{e}-2$ & $1 e-3$ & $1 e-5$ & $1 \mathrm{e}-7$ & \#succ \\
\hline f4 & 809 & 1633 & 1688 & 1758 & 1817 & 1886 & 1903 & $15 / 15$ & f16 & 120 & 612 & 2662 & 10163 & 10449 & 11644 & 12095 & $15 / 15$ \\
\hline IA-T & $2.7(4)$ & $\infty$ & $\infty$ & $\infty$ & $\infty$ & $\infty$ & $5 e 5$ & & $\mathrm{MA}$ & $1.7(1)$ & $1(3)$ & $1.8(1)$ & $0.56(0.3)$ & & & & \\
\hline & $2.2(1)$ & $\infty$ & $\infty$ & $\infty$ & $\infty$ & $\infty$ & & & & & & & 1.6 & & & & \\
\hline $\mathrm{MA}-\mathrm{CSA}$ & $2.2(3)$ & $\infty$ & $\infty$ & $\infty$ & $\infty$ & $\infty$ & $\infty 5 e 5$ & $0 / 15$ & $\mathrm{MA}-\mathrm{CSA}$ & $2.2(1)$ & $1.9(1)$ & $1.4(1)$ & $0.49(0.3)$ & $0.54(0.2)$ & $0.55(0.3)$ & $0.56(0.3)$ & ) $15 / 15$ \\
\hline$\Delta f_{\mathrm{opt}}$ & $1 \mathrm{e} 1$ & $1 \mathrm{e} 0$ & $1 \mathrm{e}-1$ & $1 e-2$ & $1 e-3$ & $1 e-5$ & $1 e-7$ & \#succ & $\Delta f_{\mathrm{opt}}$ & $1 \mathrm{e} 1$ & $1 \mathrm{e} 0$ & $1 \mathrm{e}-1$ & $1 e-2$ & $1 e-3$ & $1 e-5$ & $1 e-7$ & \#succ \\
\hline f5 & 10 & 10 & 10 & 10 & 10 & 10 & 10 & & & 5.2 & 215 & 899 & 2861 & 3669 & & 793 & $15 / 15$ \\
\hline MA- & $4.0(2)$ & $\mathbf{O}(2)$ & $1(2)$ & $1(2)$ & $1(2)$ & $1(2)$ & (2) & & MA & $24(3)$ & $2.6(2)$ & $1.6(2)$ & $0.97(0.4$ & 1) $0.94(0.3)$ & & & 15 \\
\hline & $4.2(2)$ & $8(3)$ & & & & & & & $\mathrm{R}$ & & $0.93(0$ & 2) $0.97(0.6)$ & & & & & \\
\hline $\mathrm{IA}-\mathrm{CS}$ & $\mathbf{3 . 6 ( 0 . 9 )}$ & $5.0(2)$ & $2(2)$ & $2(2)$ & $2(2)$ & 3) & 2) & $15 / 15$ & IA & $4.2(6)$ & $0.98(0.3$ & 3) $0.53(0.3)$ & $1.0(0.2)$ & 1.2 & 1.1 & $1.3(0.4)$ & $15 / 15$ \\
\hline$\Delta f_{\text {opt }}$ & $1 \mathrm{e} 1$ & $1 \mathrm{e} 0$ & $1 \mathrm{e}-1$ & $1 e-2$ & $1 e-3$ & $1 e-5$ & e-7 & \#succ & $\Delta f_{\mathrm{opt}}$ & $1 \mathrm{e} 1$ & $1 \mathrm{e} 0$ & $1 e-1$ & $1 e-2$ & $1 e-3$ & $1 e-5$ & $1 \mathrm{e}-7$ & \#succ \\
\hline f6 & 11 & 21 & 28 & 40 & 580 & 105 & 13 & $\overline{15}$ & & 103 & 378 & 3968 & 8451 & 9280 & 109 & 12 & \\
\hline IA-T & $2.2(0.9)$ & $9(0.2)$ & 0 & 7( & 4( & & & & MA & 0.92 & 5) $1.8(2)$ & $0.67(1)$ & $0.59(0.4)$ & $\mathbf{0 . 6 9}(0.4)$ & ) 0.7 & 0. & ) 15 \\
\hline & $2.5(0$ & $0(\mathrm{C}$ & ) & a & o & & & & & & $5.0(6)$ & $1.0(2)$ & $0.70(0.3)$ & & & & \\
\hline $\mathrm{A}-\mathrm{C}$ & $2.0(0.9)$ & $1.9(0.3)$ & $(0.4)$ & $1.8(0.1)$ & $1.5(0.3$ & $(0.2$ & 0.2 & $15 / 15$ & MA & $1.3(2$ & $2.4(0.1)$ & $\mathbf{0 . 6 1}(0.4)$ & $0.54(0.6)$ & $0.74(0.5)$ & $0.77(0.4)$ & 0.9 & $15 / 15$ \\
\hline$\Delta f_{\mathrm{opt}}$ & $\mid 1 \mathrm{e} 1$ & $1 \mathrm{e} 0$ & -1 & $1 \mathrm{e}-2$ & $1 \mathrm{e}-3$ & $1 e-5$ & -7 & \#succ & $\Delta f_{\mathrm{c}}$ & $\mid 1 \mathrm{e} 1$ & $1 \mathrm{e} 0$ & $1 \mathrm{e}-1$ & $1 \mathrm{e}-2$ & $1 \mathrm{e}-3$ & $1 e-5$ & $1 \mathrm{e}-7$ & \#succ \\
\hline & & 324 & 1171 & 1451 & 1572 & 1572 & 159 & 115 & & 1 & 1 & 242 & $1.0 \mathrm{e}$ & $1.2 \mathrm{es}^{5}$ & $1.2 \mathrm{e} 5$ & $1.2 \mathrm{e} 5$ & $\overline{15}$ \\
\hline A-T & $4.1(2)$ & $98(1)$ & $.93(0.5)$ & $0.86(0.4)$ & $0.82(0.3)$ & ) 0.8 & $\mathbf{0 . 8 3}(0.7)$ & 15 & 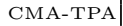 & 21) & $959(7$ & $4(62)$ & & 78 & $(0.50 .80)$ & $7 x$ & 15 \\
\hline & 5.3 & & & ) 0.5 & & & & & & & 2573 & 43) 306 & 67 & $\infty$ & $\infty$ & & \\
\hline $\mathrm{MA}-\mathrm{CSA}$ & $4.8(2)$ & $1.3(1)$ & $\mathbf{0 . 8 7}(0.8)$ & $0.80(0.9)$ & $0.80(0.8)$ & $0.80(0.7)$ & $0.86(0.6)$ & $15 / 15$ & MA- & $\mathbf{1 9}(12)$ & $2971(310$ & 03) $153(107$ & 0.8 & $(0.7) 0.83(0$ & $0.7) 0.83(0$ & $.7) 0.84(0$ & 6) $15 / 15$ \\
\hline$\Delta f_{\mathrm{opt}}$ & $\mid 1 \mathrm{e} 1$ & $1 \mathrm{e} 0$ & $e-1$ & $1 \mathrm{e}-2$ & $1 \mathrm{e}-3$ & $1 e-5$ & $1 \mathrm{e}-7$ & \#succ & $\Delta f_{\mathrm{O}}$ & $\mid 1 \mathrm{e} 1$ & $1 \mathrm{e} 0$ & $1 e-1$ & $e-2$ & $1 \mathrm{e}-3$ & $1 \mathrm{e}$ & $1 \mathrm{e}-7$ & \#: \\
\hline f8 & 75 & 273 & 33 & 372 & 391 & 410 & 422 & & & 16 & 851 & 38 & 513 & & & & \\
\hline & 4 & & & & & & & & & & & & 5 & & & & \\
\hline & $4.6(3)$ & & & & & & & 15 & & & 200 & 36) & 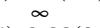 & $\infty$ & & & 15 \\
\hline A-C & $\mathbf{3 . 0}(0.8)$ & $5.1(5)$ & $5.3(4)$ & $5.4(4)$ & $5.5(3)$ & $0.1(2)$ & $0(4)$ & $15 / 15$ & MA & 3.7(1) & & $1.1(0.5)$ & 5) 0.83 & $.4) 0.80$ & $6) \mathbf{0 . 8 2}$ & 5) 0.84 & $15 / 15$ \\
\hline$\Delta f_{\text {opt }}$ & $1 \mathrm{e} 1$ & $1 \mathrm{e} 0$ & $1 \mathrm{e}-1$ & $1 \mathrm{e}-2$ & $1 \mathrm{e}-3$ & $1 e-5$ & $1 \mathrm{e}-7$ & \#succ & $\Delta f$ & $1 \mathrm{e} 1$ & $1 \mathrm{e} 0$ & $1 \mathrm{e}-1$ & $1 \mathrm{e}-2$ & $1 e-3$ & $1 \mathrm{e}-5$ & $1 \mathrm{e}-7$ & \#succ \\
\hline f9 & 35 & 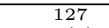 & 21 & $26:$ & 30 & & 36 & & & 41 & 115 & 1674 & 1692 & 170 & 172 & 17 & $\overline{15}$ \\
\hline A T & $5.4(2)$ & & & & & & & & A & & & 180218 & 15 & & & & \\
\hline & & & & & & & & & & & & & & & & & \\
\hline 1A- & $5.7(0.7)$ & $10(11)$ & (1) & () & $5)$ & & & 15 & MA & $1.9(1)$ & $55(150) \quad 1$ & $119(226)$ & $148(319)$ & $147(155)$ & $145(278)$ & $143(207)$ & $9 / 15$ \\
\hline$\Delta f_{\text {opt }}$ & $1 \mathrm{e} 1$ & $1 \mathrm{e} 0$ & $e-1$ & $e-2$ & $e-3$ & $e-5$ & $e-7$ & $\mathrm{cc}$ & $\Delta f$ & $1 \mathrm{e} 1$ & $1 \mathrm{e} 0$ & $1 \mathrm{e}-1$ & $1 e-2$ & $1 \mathrm{e}-3$ & $1 e-5$ & $1 e-7$ & \#succ \\
\hline & 3 & 5 & & & & & & & & & & 93 & 98 & & & & \\
\hline T & $2.5(0$. & O & & & & & & & & & & & & & & & \\
\hline & & & & & & & & & & & $052)$ & & & & & & \\
\hline CMA-CSA & $2.5(0.4)$ & $2.1(0.2)$ & $\mathbf{2 . 0}(0.2)$ & $\mathbf{2 . 0}(0.1)$ & $\mathbf{2 . 1}(0.2)$ & $1.8(0.1)$ & $1.8(0.1)$ & $15 / 15$ & MA-CSA & 4.1(1) & $\mathbf{1 3 5}(138)$ & $345(479) \quad 4$ & $426(534)$ & $535(782)$ & $519(629)$ & $507(413)$ & $6 / 15$ \\
\hline$\Delta f_{\text {opt }}$ & $1 \mathrm{e} 1$ & $1 \mathrm{e} 0$ & $1 \mathrm{e}-1$ & $1 \mathrm{e}-2$ & $1 e-3$ & $1 e-5$ & $1 \mathrm{e}-7$ & \#succ & $\Delta f$ & $1 \mathrm{e} 1$ & $1 \mathrm{e} 0$ & $1 \mathrm{e}-1$ & $1 \mathrm{e}-2$ & $1 \mathrm{e}-3$ & $1 e-5$ & $1 \mathrm{e}-7$ & \#succ \\
\hline II & 1 & 20 & & 97 & & & & & & 3 & 51 & 142 & & & & & \\
\hline & 5.1( & & & & & & & & & 3.2 & & $8.1(4$ & 4.2 & 3.8 & & & 15 \\
\hline & & & & & & & & & & & & o. & & & & & \\
\hline $\mid \mathrm{A}-\mathrm{CSA}$ & 4.9(1.0) & $4.3(0.6)$ & ) $1.3(0.2)$ & $1.1(0.2)$ & $1.00(0.1)$ & ) $0.91(0.1)$ & $\mathbf{0 . 8 8}(0$ & $15 / 15$ & MA & (3) & $13(15)$ & $4.7(0.8$ & $2.5(2)$ & $2.2(2)$ & $2.2(2)$ & $2.1(1)$ & $15 / 15$ \\
\hline$\Delta f_{\text {opt }}$ & $1 \mathrm{e} 1$ & $1 \mathrm{e} 0$ & $1 \mathrm{e}-1$ & $1 e-2$ & $1 e-3$ & $1 e-5$ & $1 \mathrm{e}-7$ & & $\Delta$. & $1 \mathrm{e} 1$ & $1 \mathrm{e} 0$ & $1 \mathrm{e}-1$ & $1 e-2$ & $1 e-3$ & $1 e-5$ & $1 \mathrm{e}-7$ & \#succ \\
\hline & & & & & & & & & & & 2. & 6.4 & & & & & \\
\hline 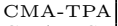 &  & $1(8)$ & & & & & & & MA-TPA & $1.3(2)$ & $10(20$ & $\infty$ & $\infty$ & 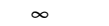 & $\infty$ & o. & 0 \\
\hline & & & & & & & & & & & ? & 1.1( & & & & & \\
\hline WA- & $0(12$ & 1(7) & (9) & 8) & & $3.5(1)$ & & $15 / 15$ & A & $2.0(2)$ & & & $\infty$ & $\infty$ & $\infty$ & $\infty 5 e 5$ & $0 / 15$ \\
\hline
\end{tabular}

Table 1: Expected running time (ERT in number of function evaluations) divided by the respective best ERT measured during BBOB-2009 in dimension 5. The ERT and in braces, as dispersion measure, the half difference between 90 and 10\%-tile of bootstrapped run lengths appear for each algorithm and target, the corresponding best ERT in the first row. The different target $\Delta f$-values are shown in the top row. \#succ is the number of trials that reached the (final) target $f_{\text {opt }}+10^{-8}$. The median number of conducted function evaluations is additionally given in italics, if the target in the last column was never reached. Entries, succeeded by a star, are statistically significantly better (according to the rank-sum test) when compared to all other algorithms of the table, with $p=0.05$ or $p=10^{-k}$ when the number $k$ following the star is larger than 1, with Bonferroni correction by the number of instances. 


\begin{tabular}{|c|c|c|c|c|c|c|c|c|}
\hline$\Delta f_{\mathrm{opt}}$ & $1 \mathrm{e} 1$ & $1 \mathrm{e} 0$ & $1 \mathrm{e}-1$ & $1 \mathrm{e}-2$ & $1 \mathrm{e}-3$ & $1 \mathrm{e}-5$ & $1 \mathrm{e}-7$ & \#succ \\
\hline f1 & 43 & 43 & 43 & 43 & 43 & 43 & 43 & $15 / 15$ \\
\hline IA-T & $6.4(1)^{\star}$ & $11(1)^{\star 3}$ & $15(2)^{\star 3}$ & $19(1)^{\star 4}$ & $24(2)^{\star 4}$ & $32(2)^{\star 4}$ & $41(2)^{\star 4}$ & $15 / 15$ \\
\hline & & $(1.0)$ & $23(3)$ & & & & & \\
\hline & $7.7(1)$ & $14(2)$ & $20(1)$ & $26(2)$ & $32(2)$ & $45(3)$ & $7(4)$ & $15 / 15$ \\
\hline$\Delta f_{\mathrm{opt}}$ & $1 \mathrm{e} 1$ & $1 \mathrm{e} 0$ & $1 \mathrm{e}-1$ & $1 e-2$ & $1 e-3$ & $1 e-5$ & e-7 & \#succ \\
\hline f2 & 385 & 386 & 387 & 388 & 390 & 391 & 393 & \\
\hline$a^{2}$ & $25(3)$ & $30(2)$ & $33(2)$ & $35(1)$ & $36(1)$ & $37(2)$ & $37(1)$ & \\
\hline & $27(4)$ & $32(4)$ & $35(2)$ & $36(2)$ & $37(2)$ & $38(3)$ & $39(2)$ & 15 \\
\hline MA-CSA & $23(2)$ & $27(2)^{\star}$ & $29(0.9)$ & $30(1)^{x}$ & $31(1.0$ & $32(2)^{\star}$ & $33(1)^{\star}$ & $15 / 15$ \\
\hline$\Delta f_{\mathrm{opt}}$ & $1 \mathrm{e} 1$ & $1 \mathrm{e} 0$ & $1 e-1$ & $1 e-2$ & $1 e-3$ & $1 e-5$ & $e-7$ & \#succ \\
\hline f3 & 5066 & 7626 & 763 & 763 & 764 & 764 & 7651 & $15 / 15$ \\
\hline MA-TPA & $8.8(5)$ & $1756(217$ & 7) $\infty$ & & & $\infty$ & $\infty 2 e 6$ & $0 / 15$ \\
\hline $\mathrm{SR}$ & $6.4(1)$ & 38( & 70 & $72(5$ & $76(41)$ & $81(36$ & ${ }^{4} 86(68)^{\star 4}$ & $15 / 15$ \\
\hline & $10(8)$ & $\infty$ & $\infty$ & $\infty$ & $\infty$ & $\infty$ & $\infty 2 e 6$ & $0 / 15$ \\
\hline$\Delta f_{\text {opt }}$ & $1 \mathrm{e} 1$ & $1 \mathrm{e} 0$ & $1 \mathrm{e}-1$ & $1 \mathrm{e}-2$ & $1 e-3$ & $1 e-5$ & $1 \mathrm{e}-7$ & \#succ \\
\hline$\rho_{1}$ & 4722 & 762 & 766 & 768 & $77 \mathrm{C}$ & 775 & $1.4 \mathrm{e} 5$ & \\
\hline & $\infty$ & $\infty$ & $\infty$ & $\infty$ & $\infty$ &  & 0 & \\
\hline & 578 & $\infty$ & $\infty$ & $\infty$ & $\infty$ & $\infty$ & $2 e$ & \\
\hline A & $\left.\right|_{\infty}$ & $\infty$ & $\infty$ & $\infty$ & $\infty$ & $\infty$ & $\infty 2 e 6$ & $0 / 15$ \\
\hline$\Delta f_{\mathrm{Op}}$ & $1 \mathrm{e} 1$ & $1 \mathrm{e} 0$ & $1 e-1$ & $1 e-2$ & e-3 & $1 e-5$ & e-7 & ucc \\
\hline & 41 & 41 & 41 & 41 & 41 & 41 & 41 & \\
\hline A & $4.3(0$ & $.9(2)$ & 9( & $\mathbf{9}(0$ & $4.9(1)$ & 9( &  & \\
\hline & & & & & & & & \\
\hline A & $4.9(1)$ & $5.8(0.9)$ & $6.0(1$ & $6.0(1$ & $.0(1)$ & .0( & $6.0(1)$ & 5 \\
\hline$\Delta f_{\mathrm{OF}}$ & $1 \mathrm{e} 1$ & $1 \mathrm{e} 0$ & $1 \mathrm{e}-1$ & $1 \mathrm{e}-2$ & -3 & $e-5$ & $1 \mathrm{e}-7$ & \# \\
\hline & 120 & 23 & & 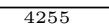 & & 6728 &  & \\
\hline & & & & & & & & \\
\hline & 1.5 & 1.9 & 2. & $.9(4)$ & $5.7(4)$ & $11(6)$ & $13(1)$ & \\
\hline MA-CSA & $1.6(0$. & $1.3(0.2)$ & $1.1(0.2)$ & $1.1(0.2)$ & $1.1(0.1$ & $1.1(0$ & 21.1( & $15 / 15$ \\
\hline$\Delta f_{\mathrm{opt}}$ & $1 \mathrm{e} 1$ & $1 \mathrm{e} 0$ & $1 e-1$ & $1 e-2$ & $1 e-3$ & $1 e-5$ & $1 e-7$ & \#succ \\
\hline & & & & & & & & \\
\hline & & & & & & & & \\
\hline & & & & & & & & \\
\hline & $1.7(1$ & $2.3(1$ & $1.7(0$. & $1.1(0$. & $1(0.3)$ & $1.1(0.4)$ & $1.0(C$ & 15 \\
\hline$f_{\mathrm{O}}$ & $1 \mathrm{e} 1$ & & & & & & & \\
\hline & & & & & & & & \\
\hline & & & & & & & & \\
\hline & & & & & & & & \\
\hline & & 3.4( & ) & $\mathbf{3 . 7}(0.3)$ & $\mathbf{3 . 8}(0$. & $3.8(0.2)$ & $3.8(0.2)$ & 15 \\
\hline$\Delta f_{\mathrm{O}}$ & $1 \mathrm{e} 1$ & & & & & & & \\
\hline & & & & & & & & \\
\hline & & & & & & & & \\
\hline & & & & & & & & \\
\hline & 3.8 & & & & & & & \\
\hline$\Delta f_{\mathrm{O}}$ & $\mid 1 \mathrm{e} 1$ & 0 & & 2 & 3 & $1 e-5$ & 7 & \\
\hline & & & & & & & & \\
\hline & & & & & & & & \\
\hline & 1.3( & 1.3 & & & & & & \\
\hline & $1.2(0.2)$ & $1.2(0$ & $1.0(0$. & $.86(C$ & b?.81( & b.74 & ) 4.76 & $135 / 15$ \\
\hline$\Delta$ & $1 \mathrm{e} 1$ & $1 \mathrm{e} 0$ & & & & & & \\
\hline & & & & & & & & \\
\hline & & & & & & & & \\
\hline & & & & 0.8 & & 0.65 ( & 0.5 & $15 /$ \\
\hline & 4.6 & 2.3( & $0 . \varepsilon$ & 0.67 & 0.63 & 6.55 & 0.50 & $15 / 15$ \\
\hline & i) & 1 & & $1 \mathrm{e}-2$ & $1 e-3$ & & & \\
\hline & & & & & & & & \\
\hline & & & & & & & & \\
\hline & & & & & & & & \\
\hline & & & & & & & & \\
\hline
\end{tabular}

\begin{tabular}{|c|c|c|c|c|c|c|c|c|}
\hline$\Delta f_{\text {opt }}$ & $1 \mathrm{e} 1$ & $1 \mathrm{e} 0$ & $1 e-1$ & $1 \mathrm{e}-2$ & $1 \mathrm{e}-3$ & $1 e-5$ & $1 \mathrm{e}-7$ & Hsuce \\
\hline & 652 & 2021 & 2751 & 3507 & 18749 & 24455 & 30201 & $\mid 15 / 15$ \\
\hline is & 4.7 & $7(3)$ & $.0(3)$ & $5.4(2)$ & & 1.3( & & \\
\hline & 4.4 & & 3) & & & & & \\
\hline$A-C$ & $3.2(4)$ & $2(3)$ & $4.0(3)$ & $4.5(1)$ & $0.93(0.4)$ & $1.1(0.6)$ & $1.3(0.7)$ & $15 / 15$ \\
\hline$\Delta f_{\text {opt }}$ & $1 \mathrm{e} 1$ & $1 \mathrm{e} 0$ & $1 e-1$ & $1 \mathrm{e}-2$ & $1 e-3$ & $1 e-5$ & $1 e-7$ & \#succ \\
\hline f14 & 75 & 239 & 304 & 451 & 932 & 1648 & 15661 & 15 \\
\hline MA- & $3.5(1)$ & $.3(0.6)$ & $2.8(0.8$ & $+3.1(0.4$ & $2.8(0.3)$ & 3.8 & & \\
\hline & & & $3.4(\mathrm{C}$ & & & & & \\
\hline & (1) & 9( & 3.7( & & & & & \\
\hline$\Delta f_{\mathrm{opt}}$ & $1 \mathrm{e} 1$ & $1 \mathrm{e} 0$ & $1 \mathrm{e}-1$ & $1 \mathrm{e}-2$ & $1 e-3$ & $1 e-5$ & $1 e-7$ & \#succ \\
\hline f15 & 303 & 1.5 & $3.1 \mathrm{f}$ & 3.2 & 3.2 & 4.5 & & \\
\hline S & 0.9 & ) 1.1( & 0.63 & & & & & \\
\hline & & & 0.5 & & & & & \\
\hline$A-C$ & 0.83 & $0.99(0.3)$ & $0.64(0$ & 0.6 & 0.6 & 0.4 & & $15 / 15$ \\
\hline$\Delta f_{\mathrm{opt}}$ & $1 \mathrm{e} 1$ & $1 \mathrm{e} 0$ & $1 e-1$ & $1 \mathrm{e}-2$ & $1 e-3$ & $1 e-5$ & $1 \mathrm{e}-7$ & \#succ \\
\hline & & & 77 & & & & & \\
\hline MA- & 1.2( & 0.78 & 0.80 & 0.6 & 0.6 & 0.6 & & \\
\hline  & 0.80 & $\vartheta^{2} .84$ & 1.1( & & & & & \\
\hline $\mathrm{MA}-$ & 1.9 & 0.6 & 0.84 & & & & & \\
\hline$\Delta$ & $1 \mathrm{e} 1$ & $1 \mathrm{e} 0$ & $1 \mathrm{e}-1$ & $1 e-2$ & $1 e-3$ & $1 e-5$ & $1 \mathrm{e}$ & $\mathrm{acc}$ \\
\hline & & & & & & & & \\
\hline 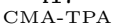 & 2.7 & & 1.5 & & & & & \\
\hline & & & & & & & & \\
\hline MA- & & 2) & 1.4 & & & & & \\
\hline$\Delta f_{\text {opt }}$ & $1 \mathrm{e} 1$ & $1 \mathrm{e} 0$ & $1 e-1$ & $1 e-2$ & $1 e-3$ & $1 e-5$ & $1 e-7$ & $\mathrm{acc}$ \\
\hline &  & & 195 & & & & & \\
\hline & & & 0.7 & & & & & \\
\hline & & & & & & & & \\
\hline MA & 0.96 & $0.72(0.1)$ & $0.81(0.4)$ & 1.1 & 0.8 & 1.1 & & \\
\hline$\Delta$ & $1 \mathrm{e} 1$ & & $1 \mathrm{e}-1$ & 10 & & & & \\
\hline & & & & & & & & \\
\hline & & & (8) & & & & & \\
\hline & & & & & & & & \\
\hline $\mathrm{M} A-$ & 221( & 3.3 & 920082 & 4) 0.5 & 2.4 & 4.5( & .0 & 15 \\
\hline$\Delta f_{\text {opt }}$ & $1 \mathrm{e} 1$ & & $1 \mathrm{e}-1$ & $1 \mathrm{e}-2$ & $1 \mathrm{e}-$ & $1 \mathrm{e}-5$ & $1 \mathrm{e}$ & \\
\hline & 8 & & & & & & & \\
\hline & & & $\alpha$ & & & & & \\
\hline & & & & & & & & \\
\hline $\mathrm{MA}-\mathrm{C}$ & $5.0(1)$ & 2.5 & 6.3 & ) 4.29 & $30 \times \frac{1}{2} \mathrm{~s}$ & 0.29 & $30 \star 30($ & ) $15 / 15$ \\
\hline$\Delta f_{\mathrm{O}}$ & $1 \mathrm{e} 1$ & & $1 \mathrm{e}$ & $1 e-2$ & & & $1 \mathrm{e}$ & \\
\hline & & & & & & & & \\
\hline & & & & & & & & \\
\hline & & & & & & & & \\
\hline$A-$ & 113( & 159 & 61) & & & & & \\
\hline$\Delta f_{\mathrm{O}}$ & $1 \mathrm{e} 1$ & $1 \mathrm{e} 0$ & $1 e-1$ & $1 \mathrm{e}-2$ & & & & \\
\hline & & & & 24 & & & & \\
\hline & & & 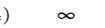 & & & & & \\
\hline & & & & & & & & \\
\hline & & & & & & & & \\
\hline$\Delta$ & 1 & & & -2 & $e-3$ & $1 e-5$ & $1 e-$ & \\
\hline & 3. & & & & & & & \\
\hline & 6.5( & & $4.8(12$ & $3.0(3)$ & & & & \\
\hline & & )$^{\star 2}$ & $0.79(0.5)$ & ) $0.74(0$ & & $0^{2} .4$ & 2) $0^{2}$ & \\
\hline & & & 13( & & & & & \\
\hline$\Delta$ & $1 \mathrm{e} 1$ & & $1 \mathrm{e}-$ & $1 \mathrm{e}-$ & $1 \mathrm{e}$ & $1 \mathrm{e}$ & & \\
\hline & 1.3 & & $5.2 \mathrm{e} 7$ & $5.2 \mathrm{e}$ & 5 . & 5 & & \\
\hline & & & & & & & & \\
\hline & & & & & & & & \\
\hline & & & & & & & & \\
\hline
\end{tabular}

Table 2: Expected running time (ERT in number of function evaluations) divided by the respective best ERT measured during BBOB-2009 in dimension 20. The ERT and in braces, as dispersion measure, the half difference between 90 and 10\%-tile of bootstrapped run lengths appear for each algorithm and target, the corresponding best ERT in the first row. The different target $\Delta f$-values are shown in the top row. \#succ is the number of trials that reached the (final) target $f_{\mathrm{opt}}+10^{-8}$. The median number of conducted function evaluations is additionally given in italics, if the target in the last column was never reached. Entries, succeeded by a star, are statistically significantly better (according to the rank-sum test) when compared to all other algorithms of the table, with $p=0.05$ or $p=10^{-k}$ when the number $k$ following the star is larger than 1, with Bonferroni correction by the number of instances. 